\title{
ANGIOEDEMA HEREDITÁRIO: CONSIDERAÇÕES SOBRE TERAPIA
}

\author{
Kélem de Nardi Chagas, Viviana Galimbert Arruk, Maria Elisa Bertocco Andrade, Dewton de \\ Moraes Vasconcelos, Michael Kirschfink, Alberto José da Silva Duarte, Anete Sevciovic Grumach* \\ Realizado no Laboratório de Investigação Médica em Dermatologia e Imunodeficiência - LIM 56 - \\ Faculdade de Medicina, Universidade de São Paulo, São Paulo, Brasil.
}

RESUMO - OBjetivos. A primeira descrição clínica completa do angioedema hereditário (HAE) foi relatada por William Osler, em 1888. As formas de angioedema com deficiência de $\mathrm{Cl}-\mathrm{INH}$ são divididas em hereditárias e adquiridas. A terapêutica pode ser direcionada aos ataques agudos ou profilaxia de novos episódios. 0 tratamento de escolha é feito através de hormônios masculinizantes, podendo também ser indicado os inibidores da ativação do cininogênio e do plasminogênio como o ácido tranexâmico ou 0 ácido e-aminocapróico e a reposição de concentrado de $\mathrm{Cl}$-INH. $\mathrm{O}$ presente estudo relata a evolução de 10 pacientes (quatro famílias) acometidos por $\mathrm{HAE}$ e as peculiaridades do tratamento utilizado em cada caso.

Métodos. Dez pacientes (I-38 anos) com HAE foram diagnosticados através de história clínica e exames laboratoriais. Os testes realizados para avaliação do sistema complemento foram: dosagem sérica de $\mathrm{Cl}-\mathrm{INH}, \mathrm{C4}$ e $\mathrm{C3}$ e ensaio hemolítico ( $\mathrm{CH} 50$ e APH50) para as vias clássica e alternativa. $\mathrm{O}$ tratamento foi indicado de acordo com a gravidade dos sintomas, idade, sexo e resposta terapêutica.

Resultados. A avaliação clínica evidenciou 4/10 pacientes com edema subcutâneo recorrente; $3 / 10$ pacientes com edema de laringe prévio e 3/10 pacientes com sintomas esporádicos. Sintomas de gravidade diferentes foram evidenciados na mesma família. A avaliação laboratorial (dosagem sérica) demonstrou: níveis de $\mathrm{Cl}-\mathrm{INH}$ diminuídos em 10/10; níveis diminuídos de $\mathrm{C4} 8 / 10$; níveis indetectáveis de $\mathrm{CH} 50$ em 3/10 e diminuídos em 6/10; níveis diminuídos de APH50 em 2/10.6/10 pacientes não receberam tratamento específico, sendo que dois deles apresentam alto risco para asfixia; uma adolescente tem sido controlada com ácido $\varepsilon$-aminocapróico, uma criança que fazia uso de danazol passou a receber ácido tranexâmico, uma paciente de 30 anos recebe oxandrolona e um homem de 38 anos está em tratamento com danazol.

Conclusão: Apesar do HAE ser causado pelo mesmo defeito e acometer membros da mesma família, diferentes critérios têm sido estabelecidos para o tratamento desses pacientes. Foram indicados diferentes esquemas terapêuticos para HAE e alguns dos pacientes puderam ser seguidos sem terapia medicamentosa.

UnITERMOS: Angioedema hereditário. Deficiência de inibidor de $\mathrm{Cl}$ esterase $(\mathrm{Cl}-\mathrm{INH})$. Tratamento.

\section{INTRODUÇÃo}

A primeira descrição clínica completa do angioedema hereditário (HAE) foi relatada por William Osler, em 1888, em cinco gerações de uma família com a doença, caracterizando a herança como autossômica dominante'! Com os estudos de Landerman et al. (1962) e Donaldson \& Evans (1963) concluiu-se que o HAE era decorrente da deficiência do inibidor de $\mathrm{Cl}$ esterase $(\mathrm{Cl}-\mathrm{INH})$ e que essa proteína era o primeiro inibidor da calicreína plasmática!. O Cl-INH é uma glicoproteína (a-globulina sérica) da família das serpinas de inibidores da protease, codificada no cromossomo II e o principal órgão responsável por sua síntese é o fígado 1,2. Várias mutações podem ser responsáveis pelo defeito; muitas

\footnotetext{
*Correspondência:

Av. Dr. Enéas de Carvalho Aguiar, n 500 - Prédio II

$3^{\circ}$ andar - Faculdade de Medicina da USP

Cerqueira César - São Paulo - SP - Brasil

CEP: 05403.000 - grumach@usp.br
}

sendo mutações pontuais ou pequenas deleções ou inserções 3,4

A maior função do $\mathrm{Cl}-\mathrm{INH}$ no corpo humano inclui a inibição direta da calicreína ativada, inibição do fator de Hageman (fator XII da cascata da coagulação) e prevenção da ativação do componente $\mathrm{Cl}$ do sistema complemento ${ }^{5}$. A diminuição da atividade de $\mathrm{Cl}-\mathrm{INH}$ permite a ativação de $\mathrm{Cl}$, com início da atividade, e consumo, de C4 e C2, resultando em diminuição de seus níveis séricos durante os ataques de $\mathrm{HAE}^{6}$.

As formas de angioedema com deficiência de $\mathrm{Cl}-\mathrm{INH}$ são divididas em hereditárias e adquiridas. As hereditárias são decorrentes da produção deficiente do inibidor com função normal (tipo I) ou da produção de quantidade normal de $\mathrm{Cl}$-INH com função alterada (tipo II), o que corresponde a $85 \%$ e I $5 \%$ dos casos, respectivamente. No tipo I, a região do gene rica em repetições Alu, sempre contém o gene defeituoso e isso explica porque pacientes do tipo I não produzem níveis normais de
$\mathrm{Cl}$-INH e não há expressão de $\mathrm{Cl}$-INH. No tipo Il, o defeito é usualmente localizado próximo do sítio ativo ARG 444 ou no exon 8. Nas formas adquiridas (AAE) ocorre uma diminuição do $\mathrm{Cl}$-INH por aumento de seu catabolismo e, freqüentemente, estão associadas com doença linfoproliferativa e com a presença de anticorpo anti-CI-INH, sem doença de base detectável; a função de $\mathrm{Cl}$-INH está moderadamente diminuída e há resistência à reposição de $\mathrm{Cl}$ purificado ${ }^{5,7-10}$. Uma redução acentuada na produção de $\mathrm{Cl} q$ é considerada como marcador das formas adquiridas de angioedema, provavelmente por degradação através de ativação induzida por complexos anti-idiotipo/idiotipo ${ }^{7}$

Clinicamente, os pacientes com HAE usualmente tornam-se sintomáticos na segunda década de vida, apresentam grande variabilidade na freqüência dos episódios e a maioria dos ataques ocorre sem qualquer razão aparente, em três sítios principais: tecido subcutâneo, tratos gastrointestinal e 


\begin{tabular}{|c|c|c|c|c|c|c|c|c|c|}
\hline Pacientes & Idade & Sexo & $\begin{array}{l}\text { Início dos } \\
\text { sintomas }\end{array}$ & Dados clínicos & $\begin{array}{l}\text { C1-INH } \\
\text { (mg/dl) }\end{array}$ & $\begin{array}{c}\mathrm{C} 4 \\
(\mathrm{mg} / \mathrm{dL})\end{array}$ & $\begin{array}{l}\text { CH } 50 \\
(\mathrm{U} / \mathrm{mL})\end{array}$ & $\begin{array}{l}\text { APH } 50 \\
\text { (U/ML) }\end{array}$ & Tratamento \\
\hline ISB & $18 a$ & $F$ & $4 a$ & Angioedema subcutâneo & 3 & 1,99 & $\overline{\text { Indet. }}$ & 65 & EACA \\
\hline FSM & $4 a 8 m$ & M & $1 \mathrm{a} 6 \mathrm{~m}$ & Dor abdominal & 15,8 & $\mathrm{Nd}$ & 54 & 68 & Semtratamento \\
\hline CSM & $30 a$ & M & $?$ & Angioedema subcutâneo & 2,54 & $\mathrm{Nd}$ & Indet. & 65 & Sem tratamento \\
\hline VFSM & $30 \mathrm{a}$ & $F$ & $8 a$ & Vômitos e diarréia & 4,3 & 2,88 & Indet. & 63 & Oxandrolona \\
\hline LFR & $6 a 10 m$ & M & $>$ I ano & Angioedema subcutâneo & 9,6 & 4,09 & 43 & 57 & Ácido tranexâmico \\
\hline ACFC & la & $F$ & 6 meses & Dor abdominal & 15 & 6,77 & 27 & 29 & Sem tratamento \\
\hline JOR & $38 a$ & M & $15 \mathrm{a}$ & $\begin{array}{l}\text { Angioedema subcutâneo e } \\
\text { de trato respiratório }\end{array}$ & 9 & 5,13 & 50 & 38 & Danazol \\
\hline COR & $38 a$ & $F$ & $?$ & Angioedema subcutâneo & 6,25 & 4,5 & 47 & 62 & Semtratamento \\
\hline $\mathrm{DaOC}$ & $16 a$ & $F$ & $3 a$ & Angioedema subcutâneo & 12,2 & 3,17 & 48 & 59 & Tratamento suspenso \\
\hline $\mathrm{DeOC}$ & $15 \mathrm{a}$ & $F$ & $?$ & Angioedema subcutâneo & 8,45 & 5 & 49 & 61 & Sem tratamento \\
\hline
\end{tabular}

EACA: ácido -aminocapróico.

$\mathrm{Nd}$ - não dosado.

Indet. - indetectável.

respiratório. O edema de laringe é a principal causa de morte associada à doença. Os ataques duram cerca de dois a cinco dias até resolverem espontaneamente ${ }^{5}$.

Os episódios de urticária aguda ou transitória e/ou angioedema acometem cerca de I 5\% a 25\% da população geral e desses, 2\% dos casos estão associados ao angioedema hereditário ${ }^{11,12,13}$. Embora sua prevalência seja baixa, as complicações como o edema de glote e a possibilidade de controle clínico dos sintomas, faz com que o seu diagnóstico seja importante, pois uma vez não diagnosticado, retarda a instituição da terapia, resultando em uma mortalidade que pode ser superior a 30\%, principalmente devido à obstrução de vias aéreas superiores ${ }^{6,10,14}$.

A terapêutica pode ser direcionada aos ataques agudos e à profilaxia de novos episódios e de procedimentos cirúrgicos ${ }^{11,15,16}$. Nas crises agudas, o tratamento de escolha consiste na administração de concentrado purificado de C1-INH ou, na falta deste, na infusão de plasma fresco congelado (PFC). A entubação oro-traqueal e outras medidas de cuidado intensivo podem ser necessárias ${ }^{17}$.

A profilaxia é indicada em pacientes com episódios muito freqüentes e/ou graves ${ }^{17}$. 0 tratamento de escolha é feito através de hormônios masculinizantes como o danazol, um andrógeno sintético atenuado, ou o stanazolol, determinando diminuição dramática tanto na freqüência quanto na gravidade dos ataques $2,11,15,17,18$. Também pode ser indicado o uso de inibidores da ativação do cininogênio e do plasminogênio como o ácido tranexâmico ou ácido $\varepsilon$-aminocapróico ${ }^{17}$.

Algumas questões permanecem com relação ao tratamento de escolha para o paciente e a resposta terapêutica obtida como: quais seriam os pacientes que receberiam terapêutica, qual o protocolo a ser seguido com relação à escolha do medicamento, o que fazer nas mulheres em fase reprodutiva e quais as outras drogas disponíveis? Com a finalidade de discutir as possibilidades terapêuticas e um possível protocolo para tratamento, o presente estudo apresenta um grupo de pacientes com HAE tipo I e que receberam abordagem terapêutica diversa mesmo em membros de uma mesma família.

\section{Métodos}

\section{Relato de casos}

Caso I: ISB, feminino, 20 anos, iniciou quadros de angioedema aos quatro anos de idade, desencadeados por trauma ou pressão. Há quatro anos, os episódios tornaram-se mais freqüentes e intensos, necessitando de internação. Recebe ácido $\varepsilon$-aminocapróico (3g/dia) com controle dos sintomas (Tabela I).

Casos 2 e 3: FSM, masculino, 4 anos e 8 meses, iniciou episódios de edema com I ano e 6 meses, precedidos de dor abdominal, desencadeado por "stress" ou trauma, apresentou múltiplas internações e não recebia tratamento anteriormente. Após o diagnóstico de HAE, foi proposta a terapêutica profilática, porém a família optou pelo não tratamento. (Figura I). Vários membros da família apresentam angioedema com manifestações graves e freqüentes, tendo sido confirmado o diagnóstico no pai (CSM) da criança (C1-INH=2,54 $\mathrm{mg} / \mathrm{dL}$ ) que também não recebe tratamento. O tio avô paterno faleceu em decorrência da crise de angioedema (Tabela I; Figura I).

Casos 4,5,6 e 7: $\mathrm{DaOC}$, feminino, 16 a IOm, apresenta episódios de angioedema desde os três anos de idade, desencadeados por trauma e que se repetiam a cada 15 dias. Referia dores articulares em MMSS e pés com melhora após aplicação de calor local. Diagnosticado colelitíase, foi submetida a colecistectomia, após ter recebido danazol e ácido tranexâmico por duas semanas e infusão de $\mathrm{Cl}-\mathrm{INH}$ precedendo o ato cirúrgico. Após a cirurgia, a paciente suspendeu o uso de medicamentos para HAE, permanecendo com crises esporádicas de edema em mãos com resolução espontânea. A mãe (COR) e a irmã (DeOC) da paciente também apresentam quadros de angioedema com crises leves e esporádicas e seu irmão apresenta episódios de dor abdominal. O tio materno, JOR (49 anos) apresenta episódios de edema desde os 15 anos, em membros e trato respiratório, com freqüência de uma por semana. É tabagista, hipertenso e diabético, tendo sido internado por 12 horas devido a edema. Recebe tratamento com danazol ( 100 mg/dia) há 10 meses com boa resposta (Tabela I; Figura 2). 
Figura I - Heredograma da família do paciente FSM

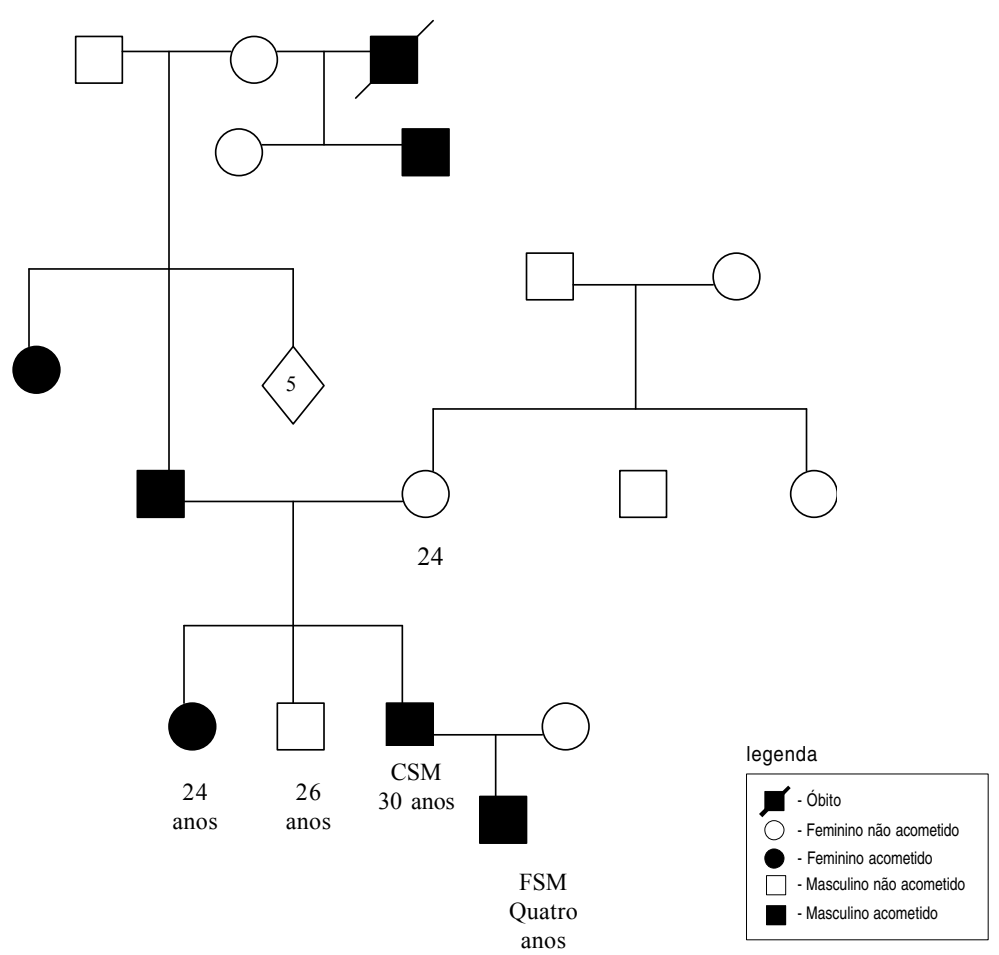

Casos 8,9 e 10: VFSN, feminino, 31 anos, iniciou quadro de angioedema aos 8 anos de idade, acometendo face e trato respiratório. Os fatores desencadeantes associados foram trauma aos 15 anos e extração dentária, assim como "stress". A paciente relata dor abdominal e discreto edema de face relacionado com frutas cítricas. Foi medicada com ácido tranexâmico, sem resposta clínica e, posteriormente, utilizou danazol, observando efeitos colaterais. Atualmente recebe oxandrolona ( $5 \mathrm{mg} / \mathrm{dia})$, com controle parcial dos sintomas. Apresentou crise intensa de edema de trato respiratório e foi medicada com concentrado de $\mathrm{Cl}$-INH. Ao ser avaliada em nosso serviço, havia tido duas gestações, apresentando crises de angioedema intenso, não medicadas (Tabela I; Figura 3). A paciente teve duas gestações, sem maiores complicações, apesar da descrição de piora dos sintomas de angioedema, por diminuição dos níveis de $\mathrm{Cl}-\mathrm{INH} H^{5,18}$. Seus dois filhos apresentam níveis diminuídos de $\mathrm{Cl}-\mathrm{INH}$ (LFR e ACFC). LFR, masculino, sete anos, apresenta crises de angioedema desde 0 primeiro ano de vida e foi medicado com danazol. Após avaliação clínica e a avaliação dos efeitos colaterais, modificou-se a terapêutica para o uso de ácido tranexâmico (Tabela I; Figura 3). ACFC, dois anos e cinco meses, apresentou episódios de dor abdominal relacionados com a ingestão de frutas cítricas. Apresenta dosagem de $\mathrm{C} 1-\mathrm{INH}$ de $15 \mathrm{mg} / \mathrm{dL}$ (Tabela I; Figura 3).

\section{Exames realizados}

Foram realizadas dosagens do nível sérico de $\mathrm{Cl}-\mathrm{INH}, \mathrm{C} 4$ e $\mathrm{C} 3$ por nefelo metria, com a utilização de anti-soro específico para os componentes do complemento (Behring, Marburg, GE) 12,19 e ensaio hemolítico para avaliação das vias clássica e alternativa do sistema complemento $(\mathrm{CH} 50$ e APH50). Em resumo, o $\mathrm{CH} 50$ e o $\mathrm{APH} 50$ foram avaliados com a determinação da habilidade do soro em lisar eritrócitos de carneiro sensibilizados com anticorpo e eritrócitos de coelho, respectivamente. Após diluição do soro, observa-se o valor correspondente a $50 \%$ de hemólise dos eritrócitos. A leitura da reação é feita em espectrofotômetro (CELM-E2 IOD) a $54 \mathrm{Inm}$ para o $\mathrm{CH} 50$ e a $412 \mathrm{~nm}$ para o APH50 19,20. Os valores de ambas as reações são obtidos após a construção 
Figura 3 - Heredograma da família da paciente VFSN

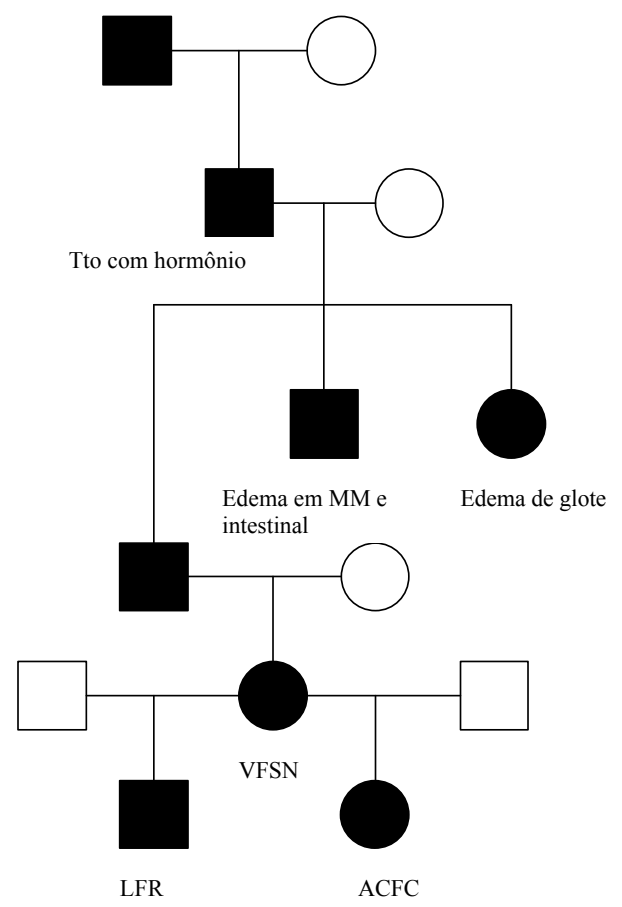

de curvas-padrão feitas simultaneamente. Os valores de normalidade foram previamente estabelecidos em nosso meio com amostras de soro normais.

\section{Resultados}

As seguintes manifestações clínicas foram observadas: edema subcutâneo recorrente (4/ 10), edema de glote anterior ( $3 / 10$ ) e sintomas esporádicos. Em membros da mesma família foram evidenciadas manifestações clínicas de gravidade variável. A avaliação laboratorial mostrou níveis séricos de $\mathrm{CI}$-INH abaixo do valor de referência (10/10), concentração de C4 diminuída (8/I0), CH50 indetectável (3/ 10) e abaixo do valor de referência (6/10) e APH50 diminuído (2/I0) (Tabela I).

Com relação ao tratamento, seis pacientes não foram medicados e, destes, dois apresentavam alto risco para asfixia. Uma adolescente recebe ácido $\varepsilon$-amicocapróico. LFR fez uso de danazol, mas devido aos efeitos colaterais, foi substituído por ácido tranexâmico, com resposta eficaz; VSFN recebe oxandrolona devido aos efeitos colaterais do uso de danazol e ao não controle dos sintomas com esta medicação. JOR faz tratamento com danazol, com bom controle clínico.

\section{Discussão}

A profilaxia a longo prazo do HAE tem sido bem sucedida, com o uso de andrógenos atenuados, como danazol e stanazolol, há mais de 30 anos e visa a prevenção das crises ${ }^{21}$. A primeira descrição de seu uso e eficácia foi feita em 1960 por Spauldin, que demonstrou que a metiltestosterona era eficaz em prevenir ataques de angioedema em uma família acometida por HAE. Sua eficácia altera a qualidade de vida dos pacientes. Atuam estimulando a síntese de $\mathrm{Cl}$-INH hepática, aumentando os níveis séricos de $\mathrm{Cl}$-INH e C45,12,24. A dose estabelecida para danazol, stanazolol e metiltestosterona é de 200 a 800 mg/dia, 2 a 12 mg/dia e 10 a 30 mg/dia, respectivamente. Os efeitos colaterais possíveis podem ser devido a: atividade hormonal residual (seborréia, acne, ganho de peso, alteração da voz, alteração menstrual, diminuição da mama, sintomas vasomotores, diminuição da libido); alteração nos níveis de lipoproteínas e a alquilação na posição 17a, que é responsável por sua hepatotoxicidade ${ }^{24}$. Mesmo com seus efeitos hepatotóxicos bem conhecidos, Sheffer et al, acompanharam pacientes com HAE, em tratamento prolongado com danazol e stanazolol, e observaram a função hepática por um período de 10 anos, demonstrando a segurança das medicações nas doses terapêuticas ${ }^{24}$. Sua maior contra-indicação está associada à gestação e à amamentação, câncer de próstata e na infância, sendo que o stanazolol parece apresentar efeitos colaterais menos intensos ${ }^{5}$.

No presente relato, quatro pacientes fizeram uso de danazol. DaOC que recebeu a medicação em altas doses antes do procedimento cirúrgico (colecistectomia) e que optou por interrupção do tratamento a seguir. JOR, tio da paciente $\mathrm{DaOC}$, encontra-se em uso de danazol $100 \mathrm{mg} / \mathrm{dia}$, há 10 meses, época em que foi encaminhado ao nosso serviço, devido à piora dos episódios de angioedema e houve controle adequado dos sintomas. VSFN apresentou efeitos colaterais com o uso de danazol, alteração da voz e ganho de peso, sendo necessária a substituição da medicação. Optouse por oxandrolona, na dose de $5 \mathrm{mg} /$ dia com melhora clínica acentuada. A oxandrolona é um 17(beta)-hidroxi- I7(alfa)-metil-2-oxa-5(alfa)androstan-3-um, semelhante a metiltestosterona, e devido a sua propriedade androgênica fraca, parece ser mais bem tolerada que os outros agentes, principalmente por mulheres ${ }^{25}$. Amin et al. descreveram o uso de oxandrolona em uma menina de 14 anos com HAE, que não estava apresentando resposta eficaz com o uso de altas doses de danazol e obtiveram controle adequado dos sintomas com esta mudança ${ }^{25}$. $O$ danazol também havia sido instituído para o controle das crises de LFR, de sete anos, entretanto, a possível repercussão sobre o crescimento indicou a mudança para ácido tranexâmico.

Os agentes anti-fibrinolíticos, como ácido tranexâmico e ácido $\varepsilon$-aminocapróico também são usados na profilaxia de ataques de HAE. ISB faz uso de ácido $\varepsilon$-aminocapróico na dose de 3g/dia, com resposta satisfatória; a opção por essa medicação foi devido à faixa etária da paciente e aos possíveis efeitos colaterais do uso de andrógenos atenuados. Estes grupos de medicamentos têm sido indicados no tratamento de crianças e em pacientes que possuem contra-indicação para o uso 
de hormônios, por exemplo, pacientes com história de tumor ${ }^{6}$. Há vários relatos do uso de agentes anti-fibrinolíticos para controle dos sintomas de angioedema, com resposta adequada e segurança, no entanto, parece que eles são melhores para a profilaxia de AAE pois, não interferem nos níveis séricos de $\mathrm{Cl}$ $\mathrm{INH}$. Embora sejam drogas de rápido metabolismo, sua eficácia é mantida ${ }^{3,5}$.

No tratamento de ataque agudo e, também, na profilaxia de procedimentos cirúrgicos, o concentrado purificado de $\mathrm{Cl}$ - INH tem sido descrito como terapêutica de escolha, uma vez que esses ataques não respondem à administração de corticosteróides, adrenalina ou anti-histamínicos, o que deve ser sempre lembrado, uma vez que estas crises assumem as características clínicas do edema de glote de etiologia alérgica| ${ }^{14,26}$.

$\mathrm{DaOC}$ fez uso do concentrado de $\mathrm{Cl}$ $\mathrm{INH}(\mathrm{I} 000 \mathrm{U})$ precedendo o procedimento cirúrgico, mas também recebeu profilaxia com danazol e ácido tranexâmico nas duas semanas que antecederam a colecistectomia. VSFN apresentou um episódio de edema de glote, na vigência do uso de danazol e fez uso do concentrado de $\mathrm{Cl}$-INH (I000 U) com boa evolução. Waytes et al relataram o uso de concentrado liofilizado para o tratamento de HAE, com aumento de nível sérico de C4 e manutenção de níveis adequados de $\mathrm{Cl}$ INH até 72 horas após a infusão. A avaliação dos pacientes que receberam infusões por quatro anos não evidenciou soro conversão para HIV ou hepatite B e C, demonstrando segurança na sua administração, assim como não houve produção de anticorpo anti-C1$\mathrm{NNH}^{22}$. Esses achados tornam este recurso - tratamento de escolha para os ataques agudos de angioedema $a^{3,5,22}$.

Ainda hoje, o plasma fresco congelado tem sido utilizado na reposição de $\mathrm{C} 1-\mathrm{INH}$, porém, o risco de transmissão de doenças virais e a presença do substrato desta enzima, responsável pelo angioedema fazem com que seu uso seja colocado em segundo plano $0^{5,12}$.

$\mathrm{Na}$ presente casuística, FSM e seu pai optaram por não receber terapêutica, embora vários membros da família houvessem falecido ou tivessem complicações decorrentes da doença. A dificuldade de compreensão dos riscos envolvidos com a conduta clínica dificultou a abordagem destes pacientes. Outra paciente, $\mathrm{DaOC}$ optou por suspender o tratamento por iniciativa própria e sua mãe e irmã também não recebem medicamentos. Deve-se ressaltar que há características diferentes nas duas famílias. No primeiro caso, as complicações relatadas nos afetados têm sido graves com evolução para o óbito antes da instituição de terapêutica, portanto, a opção pelo não tratamento pode resultar em alto risco, como mostra a experiência descrita por Bork et al. Por outro lado, a história familiar de $\mathrm{DaOC}$ mostra boa evolução com crises esporádicas sugerindo que nestes casos a evolução clínica é favorável mesmo na ausência de tratamento. Alguns critérios para a instituição de terapêutica foram sugeridos, dentre os quais, o uso de drogas apenas quando o número de crises exceder a quatro por ano. CARUGATI et al (200 I) observaram que os pacientes podem evoluir a óbito por HAE, mas também podem permanecer com manifestações leves por toda a vida.

Conclui-se, portanto, que a dificuldade de profilaxia a longo prazo para o controle do HAE está na contraposição dos benefícios de um tratamento farmacológico contínuo versus a freqüência e gravidade dos sintomas. Para cada paciente deve-se propor um esquema próprio, monitorizando-se os possíveis efeitos colaterais.

\section{Conflito de interesse: não há.}

\section{SUMMARY}

\section{THERAPEUTIC APPROACH OF HEREDITARY ANGIOEDEMA}

PURPOSE. Hereditary Angioedema was first described by William Osler in 1888 and it is caused by a hereditary or acquired deficiency of $\mathrm{Cl}$ esterase inhibitor $(\mathrm{Cl}-\mathrm{INH})$. Treatment is indicated for acute attacks or prophylaxis of angioedema which occur in the subcutaneous tissue respiratory or gastrointestinal tracts. Treatment includes attenuated androgens, inhibitors of kininogen or plasminogen, like tranexamic acid ore-aminocaproic acid and the administration of $\mathrm{Cl}-\mathrm{INH}$ concentrate. We describe the peculiarities of the treatment chosenfor 10 patients (4 families) with HAE and their evolution.

MetHODS. Ten patients (I-38years old) with HAE were diagnosed by clinical history and laboratory evaluation. The following tests were performed for the complementsystem: $\mathrm{Cl}-\mathrm{INH}$, $\mathrm{C} 4$ and $\mathrm{C} 3$ levels and hemolytic assay (CH50 and APH50) for the classic and alternative pathways. Treatment was initiated considering severity of symptoms, age, gender and therapeutic response of the patient.

RESULTS. Clinical evaluation showed: 4/10 patients with recurrentsubcutaneous edema; 31 10 with previous laryngea/edema and $3 / 10$ with sporadic symptoms. Different severity of symptoms was verified in the same family. The laboratory evaluation detected: low $\mathrm{Cl}-\mathrm{INH}$ levels (10/10); low serum C4 level (8/10); undetectable $\mathrm{CH} 50(3 / 10)$ and low $\mathrm{CH}$ 50 levels (6/10); low APH50 levels (2/10). Six out of ten patients did not receive any specific treatment and 2 of them had high risk of asphyxia. One adolescent had been controlled with $\varepsilon$-aminocaproic acid, one child had been changed from danazol to tranexamic acid, a 30 year old female patient had received oxandrolone and a 38 year old man had been treated with danazol.

CONCLUSIONS. Although HAE is caused by the same defect and affects members of the same family, various approaches have been taken to treat these patients. We observed different alternatives of prophylactic therapy for HAE, of which some did not require drug therapy. [Rev Assoc Med Bras 2004; 50(3): 314-9]

KEY wORDS: Hereditary angioedema. $\mathrm{Cl}-\mathrm{INH}$ deficiency. Treatment of HAE.

\section{REFERÊNCIAS}

I. Davis AE. CI Inhibitor gene and hereditary angioedema. In: Volanakis JE, Frank MM. The human complement system in health and disease. New York: Marcel Dekker; 1998. p.455-80.

2. Markovic SN, Inwards DJ, Frigas EA, Phyliky RP. Acquired Cl Esterase inhibitor deficiency. Ann Intern Med 2000; 132: | 44-50

3. Carugati A, Pappalardo E, Zingale LC, Cicardi M. Cl-inhibitor deficiency and angioedema. Mol Immunol 200 I; 38: I6 I-73.

4. Cicardi M, Agostoni A. Hereditary angioedema. N Engl J Med 1996; 334: I666-7.

5. Nzeako CU, Frigas E, Tremaine WJ. Hereditary angioedema. Arch Intern Med 2001; 161:2417-29.

6. Nzeako CU, Frigas E, Tremaine WJ. Hereditary angioedema as a cause of transient abdominal pain. J Clin Gastroenterol 2002; 34:54-6I.

7. D'Incan M, Tridon A, Ponard D, DumestrePérard C, Bouedec MCFL, BÉTAIL G, Souteyrand $P$, Caillaud D. Acquired angioedema with $\mathrm{Cl}$ inhibitor deficiency: is the distinction between type I and type II still relevant? Dermatology 1999; 199:227-30.

8. Walsecchi R, Reseghetti A, Pansera B, Di 
Landro A. Autoimmune Cl inhibitor deficiency and angioedema. Dermatology 1997; 195:169-72.

9. Cugno M, Cicardi M, Agostoni A. Activation of the contact system and fibrinolysis in autoimmune acquired angioedema: a rationale for prophylatic use of tranexamic acid. J Allergy Clin Immunol 1994; 93:870-6.

10. Cicardi M, Agostoni A. Hereditary and acquired $\mathrm{Cl}$-inhibitor deficiency: biological and clinical characteristics in 235 patients. Medicine 1992; 71:206-15.

I I. Zacharisen MC. Pediatric urticaria and angioedema. Immunol Allergy Clin North Am 1999; 19:363-83.

12. Moschione-Castro APB, Jacob CMA, Pastorino AC, Kirschfink M, Grumach AS. Angioedema hereditário: aspectos clínico-laboratoriais de sete casos. Rev Hosp Clín Fac Med São Paulo 1998; 53:21-5.

13. Elnick MD. Hereditary angioedema. South Med J 1992; 85: 1084-8.

14. Bork K, Siedlecki K, Bosch S, Schopf RE, Kreuz $W$. Asphyxiation by laryngeal edema in patients with hereditary angioedema. Mayo Clin Proc 2000; 75:349-54.

15. Balsa RE. Edema angioneurótico Hereditario. Arch Argent Dermatol 1997; 47:31-4I.
16. Karlis V, Glickman RS, Stern R, Kinney L. Hereditary angioedema: case report and review of management. Oral Surg Oral Med Oral Pathol Oral Radiol Endod 1997; 83:462-4.

17. Heymann WR. Acquired angioedema. J Am Acad Dermatol 1997; 36(4):6 I I-5.

18. Nielsen EW, Gran JT, Straume B, Mellbey OJ, Johansen HT, Mollnes TE. Hereditary angiooedema: new clinical observations and autoimmune screening, complement and kallikrein-kinin analises. J Intern Med 1996; 239:119-30

19. Waage Nielsen E, Thidemann Johansen $H$, Hadasen K, Wuillemin W, Hack CE, Mollnes TE. Activation of complement, coagulation, fibrinolytic and kallikrein-kinin systems during attacks of hereditary angioedema. Scand J Immunol 1996; 44:185-92.

20. Joiner KA, Hawuger A, Gelfand JA. A study of optimal reaction conditions for an assay of the human alternative complement pathway. Am J Clin Pathol 1983; 79:65-72.

21. Cicardi M, Bisiani G, Cugno M, Späth P, Agostoni A. Autoimmune $\mathrm{Cl}$ Inhibitor deficiency: report of eight patients. Am J Med 1993; 95:169-75.
22. Waytes AT, Rosen FS, Frank MM. Treatment of hereditary angioedema with a vapor-heated $\mathrm{Cl}-\mathrm{INH}$ concentrate. N Engl J Med 1996; 334:1630-4.

23. Cicardi M, Castelli R, Zingale LC, Agostoni A. Side effects of long-term prophylaxias with attenuated androgens in hereditary angioedema: Comparison of treated and untreated patients. J Allergy Clin Immunol 1997; 99:194-6.

24. Sheffer AL, Fearon DT, Austen KF. Hereditary angioedema: a decade of management with stanazolol. J Allergy Clin Immunol 1987; 80:855-60.

25. Amain J, Barakat MD, Anthony J, Castaldo MPA. Successful use of oxandrolone in the prophylaxis of hereditary angioedema: a case report. Pediatr Asthma Allergy Immunol I999; 13:189-93.

26. Jensen NF, Weiler, JM. CI Esterase inhibitor deficiency, airway compromise, and anesthesia. Anesth Analg 1998; 87:480-8.

Artigo recebido: 09/05/2003 Aceito para publicação: 17/09/2003 